\title{
climateStability: AN R PACKAGE TO ESTIMATE CLIMATE STABILITY FROM TIME-SLICE CLIMATOLOGIES
}

\author{
HANNAH L. OWEnS ${ }^{1,2, *}$ AND Robert P. GuRALNICK ${ }^{1}$ \\ ${ }^{1}$ Florida Museum of Natural History; University of Florida; Dickinson Hall, Gainesville, FL, \\ 32611, USA; ${ }^{2}$ Center for Macroecology, Evolution, and Climate; Copenhagen University; \\ Universitetsparken 15, Bld. 3, 2nd floor DK-2100 Copenhagen, Denmark; \\ *Corresponding author: hannah.owens@gmail.com
}

\begin{abstract}
As continental and global-scale paleoclimate model data become more readily available, biologists can now ask spatially explicit questions about the tempo and mode of past climate change and the impact of those changes on biodiversity patterns. In particular, researchers have focused on climate stability as a key variable that can drive expected patterns of richness, phylogenetic diversity and functional diversity. Yet, climate stability measures are not formalized in the literature and tools for generating stability metrics from existing data are nascent. Here we define "deviation" of a climate variable as the mean standard deviation between time slices over time elapsed; "stability" is defined as the inverse of this deviation. Finally, climate stability is the product of individual climate variable stability estimates. We also present an $R$ package, climateStability, which contains tools for researchers to generate climate stability estimates from their own data.
\end{abstract}

\section{INTRODUCTION}

Many hypotheses have been offered to explain geographic patterns of biodiversity from evolutionary and ecological perspectives (MacArthur, 1984). It has often been suggested that decreased extinction and increased speciation rates have led to an accumulation of species in the tropics (Rohde, 1992; Rolland, et al. 2014). Proposed drivers of this diversification rate pattern include reduced seasonality and long-term climate stability in the tropics, which, in addition to reducing extinction due to stochastic climate events, may have allowed species to specialize in narrow abiotic ecological niches and allowed a greater diversity of species to accumulate. Outside of the tropics, pockets of relatively stable conditions, introduced by Heusser in 1955 as refugia, may have provided species with the means to escape climate-driven extinctions in the past. Conversely, in temperate climates, seasonality and cyclical warming and cooling may have both driven species to remain generalists to accommodate varying conditions (Gouveia, et al. 2013; Sandel, et al. 2011) and extirpated those species that could not accommodate such climatic stochasticity. Yet, it has also been suggested that climate instability has driven the formation of biodiversity hotspots in some systems, particularly as it pertains to allopatric speciation during glacial cycles (Mayr and O'Hara, 1986; Nakazawa and Peterson, 2015).

With advances in climate modeling, more and higher-resolution climate data are available for bi- ologists to infer how past climate change may have influenced modern biodiversity patterns. Recently, Loarie and colleagues (2009) proposed "climate velocity" (the speed an individual would have to travel to track analogous climatic conditions) as a useful way to estimate potential climate change impacts on the position of organisms' ranges. A simplified version of this metric has also become common: dividing the difference between past and modern climate variable values (e.g. average temperature, average yearly rainfall) by the time elapsed between the two values (e.g. Ornelas, et al. 2015). An even simpler metric of climate change is "climate anomaly", the difference between climate variables between time slices of interest (e.g. Feng, et al. 2017). However, while these metrics are useful for estimating the degree of climate change between two time points, none adequately characterizes the stability of climates over time.

With the advent of Paleoview (Fordham, et al. 2017) and other paleoclimate assembly tools and datasets (e.g. Brown, et al. 2018; Fick and Hijmans, 2017), as well as expanded computing capacity to process large climatological datasets, time slice climatologies from climate model data are now more accessible than ever. There is also an increasing wealth of uneven time slice data from empirical inferences of climate based on geologic or archeological proxies. ${ }^{1}$ While the time-frame and exact measure of climate

1https://www.ncdc.noaa.gov/paleo-search/ 
stability may vary according to the research question, we propose here a clear definition of climate stability, as well as provide an implementation of our metric to facilitate macroecological analyses predicated on climate stability hypotheses. For each climate variable of interest, deviation through time is calculated as the mean of standard deviations between time slices divided by the time elapsed between time slices; "stability" is defined as the inverse of deviation through time. Finally, climate stability is the product of individual variable stability estimates that have been re-scaled between 0 and 1 . Herein, we present an $\mathrm{R}$ package, climateStability, with functions to perform these calculations and visualize the results, as well as providing a set of world-wide climate temperature and precipitation deviation-through-time layers over the last $21 \mathrm{kybp}$, from which climate stability can be quickly estimated. These data may then facilitate empirical tests of two key hypotheses in bioeographic theory: 1) Diversity results from a cradle-like environment where extinction resulting from climatic stocasticity is infrequent, and 2) Diversity results from repeated isolation events, often driven by climate change. We also discuss the challenges with temporal grain and interpretation when developing climate stability datasets.

General package structure and functionality. The climateStability R package allows users to:

1. Calculate stability for individual climate variables over even or uneven time slices.

2. Calculate overall climate stability from individual climate variable stability estimates.

3. Visualize resulting estimates either in geographic space, or as a graph showing the relationship between latitude and stability.

For the climateStability workflow, the user first produces rasters depicting estimated deviation through time for a single climate variable of interest by loading a series of climatology estimates (as raster files) and stipulating the time elapsed between each time slice (either as a single value if the time elapsed between climatologies is the same for all, or as a vector of values specifying the time elapsed between climatologies in order). This is repeated for each climate variable of interest. Next, for each variable, the user then calculates the inverse of the deviation and rescales the result to a scale between 0 and 1, in order to render comparable the stability estimates for each variable. Overall climate stability can then be estimated by taking the product of stability estimates for each individual variable and rescaling the result to a scale between 0 and 1. Finally, climateStability provides two functions to graph the relationship between a given latitudinal band and stability (for single variables or overall climate) - one for raw latitude $\left(-90^{\circ}\right.$ to $\left.90^{\circ}\right)$, and one for the absolute value of latitude $\left(0^{\circ}\right.$ to $90^{\circ}$ ). We have also provided two raster layers of deviation estimates for global precipitation and temperature from 21 to $1 \mathrm{kypb}$, as described below, and a vignette describing the basic climateStability workflow (Appendix 2).

\section{Methods}

Climate data. Paleoclimate estimates were derived from the TRaCE2 $1 \mathrm{ka}$ experiment (Liu, et al. 2009; Liu, et al. 2014; Otto-Bliesner, et al. 2014) implemented using the Community Climate System Model, version 3 (CCSM3; Collins, et al. 2006; Otto-Bliesner, et al. 2006; Yeager, et al. 2006). More details on these data are described by Fordham and colleagues (2017). Briefly, the CCSM 3 is a mathematical model that simulates coupled atmosphere and ocean climatic conditions given a particular set of parameters, such as those from the TraCE21ka experiment, which sought to simulate the rapid global climate changes of the last 21,000 years. The model produces monthly snapshot estimates of the simulated climate over the duration of a particular run (20,000 years, in the case of TraCE21ka), which can then be processed into climatic means at a given time point. Using Paleoview, version 1.1 (Fordham, et al. 2017) we generated 20 100-year climatological means for 1,000-year time slices between 1 and $21 \mathrm{kypb}$ for annual precipitation and mean temperature at a resolution of 2.5 degrees. We also selected eight climatologies (from 1, 2, 3, 4, 5, 10, 15, and $21 \mathrm{kybp}$ ) from the original set of 20 to simulate an unevenly-sampled time slice series. This was done to demonstrate the properties of our climate stability measurement under such conditions as might exist when a researcher wishes to incorporate deeper-time slices or time slices based on empirical climate inferences. We chose these specific time slices to simulate a "pull of the present" scenario where data becomes scarcer deeper in time.

Calculation. For each time interval, we calculated the standard deviation of a given variable at the beginning and end of the interval and divided the result by the length of the interval to quantify deviation 
over time for each time slice, then averaged the result across all time slices. We then took the inverse of this metric and scaled it to between 0 and 1 to estimate the relative stability for each variable. Finally we multiplied the stability estimate for mean annual precipitation by the stability estimate for mean annual temperature to estimate overall climate stability over the last 21,000 years. We performed these calculations both for our original climate dataset and the thinned dataset (hereafter referred to as the "even dataset" and "uneven dataset", respectively), and plotted the results as a both as raster maps and linear graphs of the relationship between stability versus latitude, all in the R programming platform (R Core Team, 2013). Code for these functions, as well as the temperature and precipitation deviation-through-time layers we produced can be downloaded as the $\mathrm{R}$ package $\mathrm{cli}$ mateStability ${ }^{2}$. Code for the analyses as described herein is available as Appendix 3.

\section{RESULTS}

Over the last 21,000 years, temperature has been most stable in the tropics and decays as latitude increases, but the patterns can be complex (e.g. low temperature stability in the Amazon Basin over the time period examined; Fig. 1). Other notable areas of low temperature stability are found in northern North America, Europe, and Asia as well as central Australia; high temperature stability areas include sub-Saharan Africa and the Indian subcontinent. There is no consistent pattern between precipitation stability and latitude in this dataset (Fig. 2); instead, terrestrial precipitation stability appears highest in central Asia and Antarctica, whereas marine precipitation stability appears highest in areas with cold ocean currents in the temperate southern Pacific and Atlantic (Fig. 1). Overall climate stability appears more strongly dictated by precipitation stability than by temperature (Figs. 1 and 2).

Generally, the uneven time slice dataset estimated congruent patterns of temperature, precipitation, and overall stability compared to the even dataset (Supplementary Figure 1). The most dramatic disagreements between the even and uneven datasets are found in the temperature stability estimates (Supplementary Figure 2) - the even dataset estimates higher stability in the tropics, whereas the uneven dataset estimates higher stability in the temperate zones, especially in the southern hemisphere. These

${ }^{2}$ https://github.com/hannahlowens/climateStability
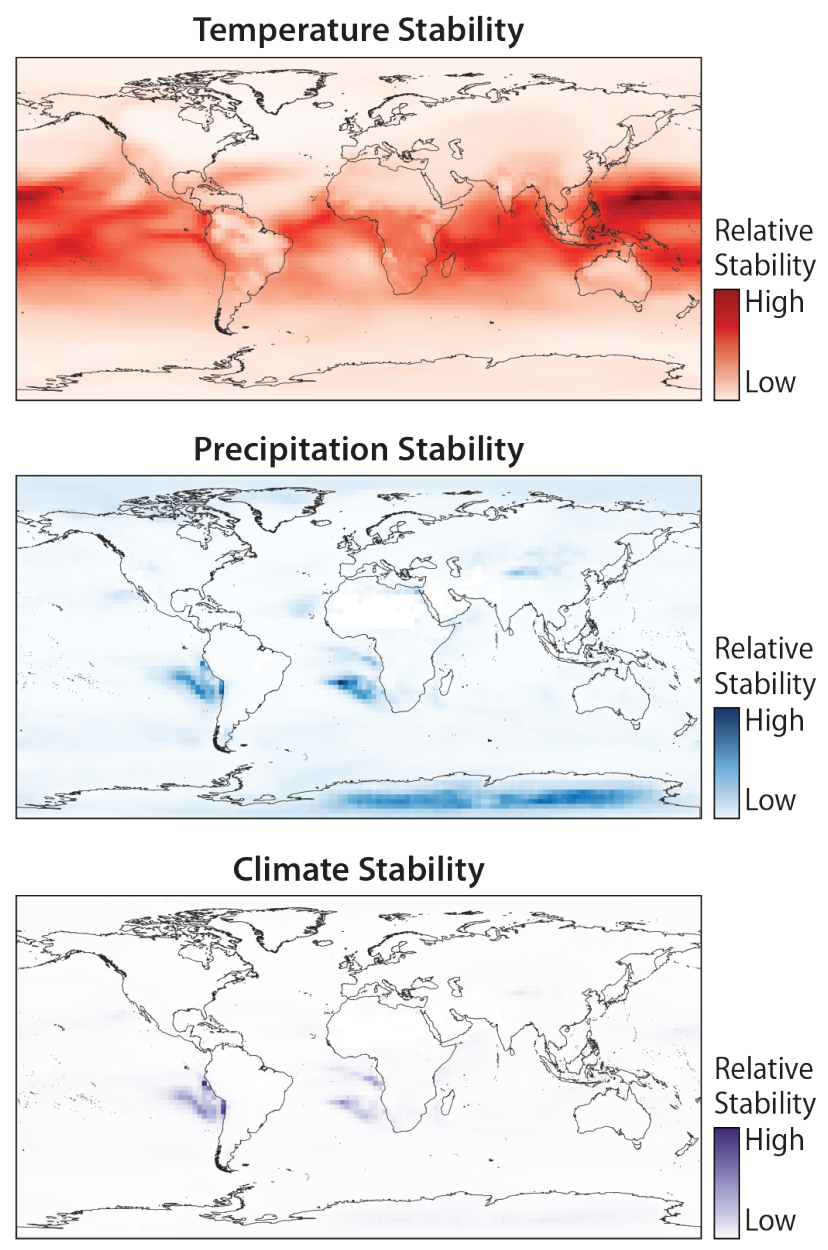

Figure 1. Relative temperature, precipitation, and climate stability based on even dataset. Darker colors indicate higher stability.

trends are also reflected in the mean stability by latitude plots (Fig. 2).

\section{Discussion}

Our estimated geographic patterns of temperature stability largely reflect glaciation patterns over the past 21,000 years, conforming to a latitudinal gradient predicted in macroecological and biogeographic theory (Slobodkin and Sanders, 1969). Conversely, patterns of precipitation stability appear to follow atmospheric circulation patterns, with areas of lowest stability occurring in areas of atmospheric upwelling and highest stability occurring with downwelling areas. Temperature and precipitation do not play the same role in defining species' suitable abiotic niches across the tree of life (Araujo and Guisan, 2006); therefore, it may be informative to consider these variables separately when assessing the degree to which climate stability influences species diversity. Still, our metric of climate stability provides an 

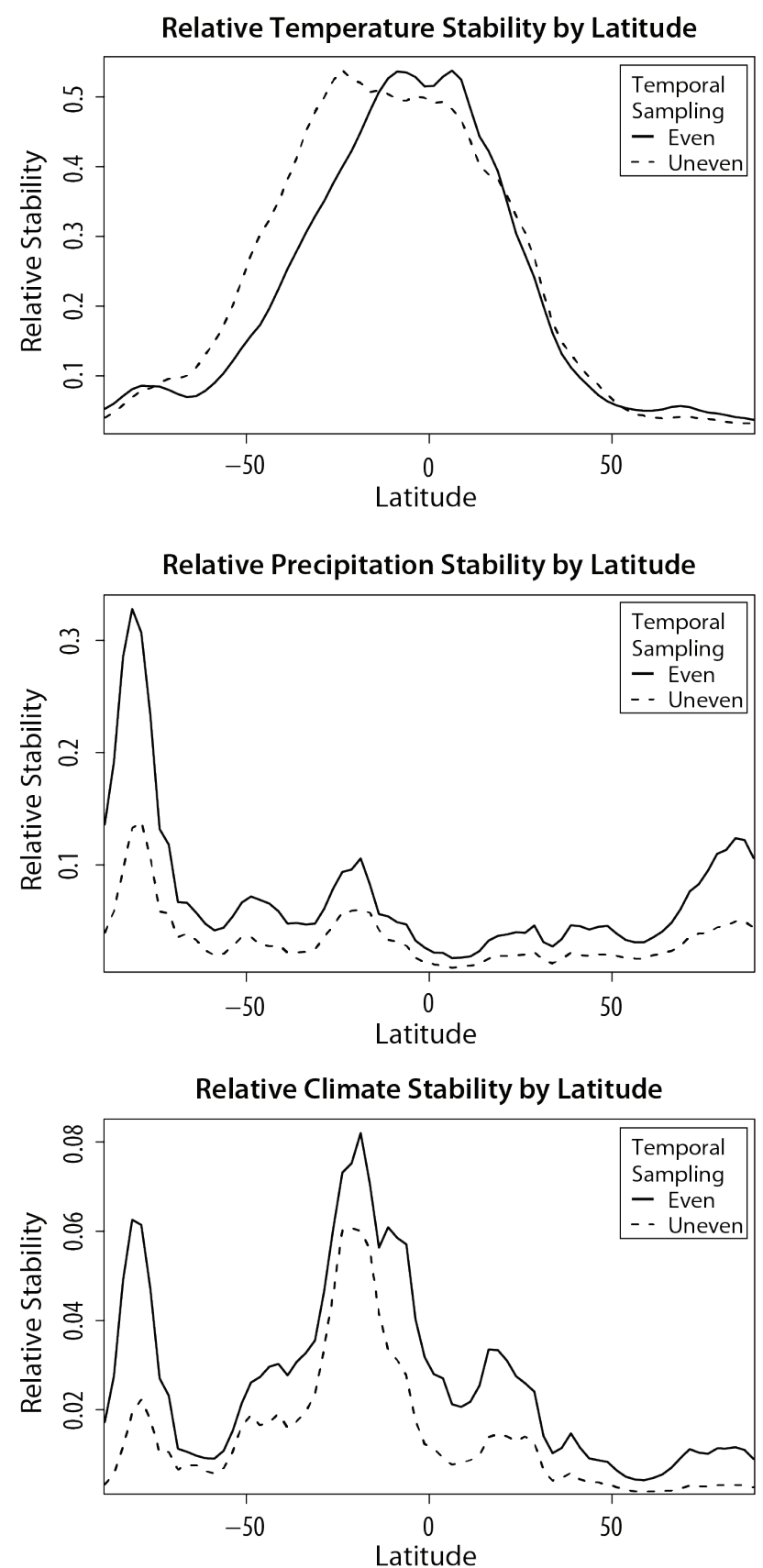

Figure 2. Plots of temperature, precipitation, and climate stability versus latitude. Solid line: even dataset; dashed line: uneven dataset.

intuitive means of quantifying a key macroecological driver of distributions of biodiversity.

Our larger aim with this contribution has been to provide a more formal basis for generating climate stability measures. Stability has been addressed more and more as a key hypothesis in the biodiversity literature, but there have been multiple types of measurements and underlying data used (Garcia et al., 2014). While these uses are often reasonable in the con- text of research questions, it can be hard to evaluate across studies, and different metrics can support very different conclusions regarding how climate change affects assemblages, species, and populations at local and regional scales (Garcia et al., 2014). We limit our definition here to climate stability. Climate stability or lack thereof can be a driver of habitat (e.g. vegetation) stability or instability, which is more likely to be the ultimate driver of species' extinction or persistence in the face of climate change (Ashcroft, 2010). Clear labeling of the stability metric employed will help tremendously not only to understand broader past trends, but also to make predictions about the effects of climate change in the future.

As well, we urge reporting of temporal grain when considering climate stability metrics - stability or lack thereof is determined in part by temporal smoothing imposed by sampling grain. Our even dataset used 1,000 year time slices, but over longer timescales than the Late Pleistocene and Holocene, the lack of fine-grain and even climatological time-slices makes the inference of stability more challenging. As our simulated uneven dataset illustrates, estimates of climate stability may be greatly influenced by the rate of variation through time. Generally, we would expect a dataset with fewer time slices to estimate lower climate stability than a dataset with more time slices due to temporal smoothing. However, the uneven dataset generally estimated higher temperature stability than the even dataset at all but the lowest latitudes (Fig. 2); when these differences mapped, it is clear that the uneven dataset estimated higher temperature stability particularly at middle latitudes (Supplemental Fig. 2). Given that the uneven dataset was largely biased toward modern time slices, this suggests that the rate of change in temperature decelerates at middle and high latitudes as we approach the present.

Finally, while the example provided herein used data from a single climate model under a single set of conditions, we recommend estimating climate stability with data from multiple climate models, when available. Different climate models have different sensitivities to climatic conditions, and may differ in their estimates of local and/or extreme phenomena-this is true of both past and future climate simulations. To generate particularly robust estimates of climate stability, researchers may want to either perform additional analyses to test how sensitive their results are to the climate model used, or use data 
from ensemble model outputs such as those from the Paleoclimate Modelling Intercomparison Project $(\mathrm{PMIP})^{3}$ and Coupled Model Intercomparison Project (CMIP) $)^{4}$.

While our climate stability metric is not completely robust to the effects of uneven time slices, it consistently identifies areas of high and low stability regardless of temporal resolution and distribution of climate data. We hope the contribution presented here precipitates a step forward from the qualitative assessment that the tropics are generally climatically stable to a more quantitative assessment of where the tropics are stable, over what time periods, and at what temporal and spatial resolution. This will facilitate more thorough examination of the role of climate stability in driving diversity patterns, which may, in turn, provide critical calibration needed for understanding how continuing and accelerating climate instability may ultimately structure future biodiversity.

\section{SOFTWARE AVAILABILITY}

Software available on CRAN ${ }^{5}$.

Install the latest release in $\mathrm{R}$ as install.packages("climateStability"), and latest development version as devtools:: install_github(“hannahlowens/climateStability”).

\section{SUPPLEMENTARY MATERIALS}

Supplementary Materials cited in the text are available at https://doi.org/10.17161/1808.28080.

\section{License: GNU General Public License v. 3.0}

\section{ACKNOWLEDGMENTS}

We thank B. F. Oliviera, collaboration with whom was the inspiration for this project. We were supported by the U.S. National Science Foundation (NSF) Division of Environmental Biology (DEB) grant \# 1541500.

\section{COMPETING INTERESTS}

The authors have declared that no competing interests exist.

\section{REFERENCES}

Araujo, M.B., and A. Guisan. 2006. Five (or so) challenges for species distribution modelling. J. Biogeogr. 33:1677-88.

\footnotetext{
${ }^{3}$ https://pmip.lsce.ipsl.fr/

${ }^{4}$ https://esgf-node.llnl.gov/projects/esgf-llnl/

${ }^{5}$ https://cran.r-project.org/web/packages/climateStability/index.html
}

Ashcroft, M.B. 2010. Identifying refugia from climate change. J. Biogeogr. 37: 1407-1413.

Brown, J., Hill, D.J., Dolan, A.M., Carnaval, A.C., and A.M. Haywood. 2018. PaleoClim, high spatial resolution paleoclimate surfaces for global land areas. Sci. Data 5: 180254.

Collins, W.D., Bitz, C.M., Blackmon, M.L., Bonan, G.B., Bretherton, C.S., Carton, J.A., Chang, P., Doney, S.C., Hack, J.J., Henderson, T.B. and J.T. Kiehl. 2006. The community climate system model version 3 (CCSM3). J. Clim. 19: 2122-2143.

Feng, G., Ma, Z., Benito, B.M., Normand, S., Ordonez, A., Jin, Y., Mao, L., and J.-C. Svenning. 2017. Phylogenetic age differences in tree assemblages across the northern hemisphere increase with long-term climate stability in unstable regions. Glob. Ecol. Biogeogr. 26: $1035-1042$.

Fick, S.E. and R.J. Hijmans, 2017. Worldclim 2: New $1-\mathrm{km}$ spatial resolution climate surfaces for global land areas. Int. J. Climatol. 37: 4302-4315.

Fordham, D.A., Saltré, F., Haythorne, S. , Wigley, T.M., Otto-Bliesner, B.L., Chan, K.C. and B.W. Brook. 2017. PaleoView: a tool for generating continuous climate projections spanning the last 21,000 years at regional and global scales. Ecography 40: 1348-1358.

Garcia, R.A., Cabeza, M., Rahbek, C., and M.B. Araújo. 2014. Multiple dimensions of climate change and their implications for biodiversity. Science 344: 1247579.

Gouveia, S.F., Hortal, J., Cassemiro, F.A.S., Rangel, T. F. and J.A.F. Diniz-Filho. 2013. Nonstationary effects of productivity, seasonality, and historical climate changes on global amphibian diversity. Ecography 36: 104-113.

Heusser, C.J. 1955. Pollen profiles from the Queen Charlotte Islands, British Columbia. Can. J. Bot. 33: 429449.

Liu, Z., Otto-Bliesner, B.L., He, F., Brady, E.C., Tomas, R., Clark, P.U., Carlson, A.E., Lynch-Stieglitz, J., Curry, W., Brook, E. and D. Erickson. 2009. Transient simulation of last deglaciation with a new mechanism for Bølling-Allerød warming. Science 325: 310-314.

Liu, Z., Lu, Z., Wen, X., Otto-Bliesner, B.L., Timmermann, A. and K.M. Cobb. 2014. Evolution and forcing mechanisms of El Niño over the past 21,000 years. Nature 515: 550.

Loarie, S.R., Duffy, P.B., Hamilton, H., Asner, G.P., Field, C.B., and D.D. Ackerly. 2009. The velocity of climate change. Nature 462: 1052-1055.

MacArthur, R.H. 1984. Geographical ecology: Patterns in the distribution of species. Princeton University Press, Princeton, New Jersey. 269 pp. 
Otto-Bliesner, B.L., Tomas, R., Brady, E.C., Ammann, C., Kothavala, Z. and G. Clauzet. 2006. Climate sensitivity of moderate-and low-resolution versions of CCSM3 to preindustrial forcings. J. Clim. 19: 25672583.

Mayr, E. and R.J. O’Hara. 1986. The biogeographic evidence supporting the Pleistocene forest refuge hypothesis. Evolution 40: 55-67.

Nakazawa, Y. and A.T. Peterson. 2015. Effects of climate history and environmental grain on species' distributions in Africa and South America. Biotropica 47: 292-299.

Neiva, J., Paulino, C., Nielsen, M.M., Krause-Jensen, D., Saunders, G.W., Assis, J., Bárbara, I., Tamigneaux, É., Gouveia, L., Aires, T., Marbà, N., Bruhn, A., Pearson, G.A. and E.A. Serrão. 2018. Glacial vicariance drives phylogeographic diversification in the amphi-boreal kelp Saccharina latissima. Sci. Rep. 8: 1112.

Nevado, B., Contreras-Ortiz, N., Hughes, C. \& Filatov, D.A. (2018) Pleistocene glacial cycles drive isolation, gene flow and speciation in the high-elevation Andes. New Phytol. 219: 779-793.

Otto-Bliesner, B.L., Russell, J.M., Clark, P.U., Liu, Z., Overpeck, J.T., Konecky, B., Nicholson, S.E., He, F. and Z. Lu. 2014. Coherent changes of southeastern equatorial and northern African rainfall during the last deglaciation. Science 346: 1223-1227.

Ornelas, J.F., S.G.D. León, C. González, Y. Licona-Vera, A.E. Ortiz-Rodriguez and F. Rodríguez-Gómez. 2015. Comparative palaeodistribution of eight hummingbird species reveal a link between genetic diversity and quaternary habitat and climate stability in Mexico. Folia Zool. 64: 245-258.
Owens, H.L., Lewis, D.S., Dupuis, J.R., Clamens, A.L., Sperling, F.A., Kawahara, A.Y., Guralnick, R.P. and F.L. Condamine. 2017. The latitudinal diversity gradient in New World swallowtail butterflies is caused by contrasting patterns of out-of-and into-the-tropics dispersal. Glob. Ecol. Biogeogr. 26: 1447-1458.

R Core Team. 2013. R: A language and environment for statistical computing. R Foundation for Statistical Computing, Vienna, Austria. ${ }^{6}$

Rangel, T.F., Edwards, N.R., Holden, P.B., Diniz-Filho, J.A.F., Gosling, W.D., Coelho, M.T.P., Cassemiro, F.A., Rahbek, C. and R.K. Colwell. 2018. Modeling the ecology and evolution of biodiversity: Biogeographical cradles, museums, and graves. Science 361: eaar5452.

Rohde, K. 1992. Latitudinal gradients in species diversity: the search for the primary cause. Oikos 65: 514-527.

Rolland, J., Condamine, F.L., Jiguet, F. and H. Morlon. 2014. Faster speciation and reduced extinction in the Tropics contribute to the mammalian latitudinal diversity gradient. PLOS Biol. 12: e1001775.

Sandel, B., Arge, L., Dalsgaard, B., Davies, R.G., Gaston, K.J., Sutherland, W.J. and J.-C.Svenning. 2011. The influence of late Quaternary climate-change velocity on species endemism. Science 334: 660-664.

Slobodkin, L.B., and H.L. Sanders. 1969. On the contribution of environmental predictability to species diversity. Brookhaven Symp. Biol. 22:82-95.

Yeager, S.G., Shields, C.A., Large, W.G. and J.J. Hack. 2006. The low-resolution CCSM3. J. Clim. 19: 25452566.

${ }^{6 h t t p: / / w w w . R-p r o j e c t . o r g / ~}$ 